\title{
Joint modeling of the two-alternative multidimensional forced-choice personality measurement and its response time by a Thurstonian $\mathrm{D}$-diffusion item response model
}

\author{
Kyosuke Bunji ${ }^{1,2}$ (i) $\cdot$ Kensuke Okada ${ }^{1}$ (i)
}

Published online: 11 May 2020

(C) The Psychonomic Society, Inc. 2020

\begin{abstract}
The two-alternative multidimensional forced-choice measurement of personality has attracted researchers' attention for its tolerance to response bias. Moreover, the response time can be collected along with the item response when personality measurement is conducted with computers. In view of this situation, the objective of this study is to propose a Thurstonian D-diffusion item response theory (IRT) model, which combines two key existing frameworks: the Thurstonian IRT model for forced-choice measurement and the D-diffusion IRT model for the response time in personality measurement. The proposed model reflects the psychological theories behind the data-generating mechanism of the item response and response time. A simulation study reveals that the proposed model can successfully recover the parameters and factor structure in typical application settings. A real data application reveals that the proposed model estimates similar but still different parameter values compared to the original Thurstonian IRT model, and this difference can be explained by the response time information. In addition, the proposed model successfully reflects the distance-difficulty relationship between the response time and the latent relative respondent position.
\end{abstract}

Keywords Item response theory $\cdot$ Response time $\cdot$ Diffusion model $\cdot$ Thurstonian IRT

The Likert scale (Likert, 1932) is widely used to measure respondents' psychological characteristics. However, it is also known that the data obtained from a Likert scale tend to be affected by response biases (for more details and examples, see e.g., Chen, Lee, \& Stevenson, 1995; Ferrando \& Lorenza-Seva, 2010; He, Bartram, Inceoglu, \& Vijver, 2014; Kam \& Meyer, 2015; Liu, Harbaugh, Harring, \& Hancock, 2017; Phelps, Schmitz, \& Boatright, 1986; Rammstedt \& Farmer, 2013; Salgado, 2016; Thunholm 2001; Usami,

This research was supported in part by JSPS KAKENHI, grant numbers $17 \mathrm{~J} 07674$ (K.B.) and 17H04787 (K.O.)

Electronic supplementary material The online version of this article (https://doi.org/10.3758/s13428-019-01302-5) contains supplementary material, which is available to authorized users.

Kyosuke Bunji

bunji@p.u-tokyo.ac.jp

1 Graduate School of Education, The University of Tokyo, Hongo 7-3-1, Bunkyo-ku, Tokyo, Japan

2 Japan Society for the Promotion of Science, Tokyo, Japan
Sakamoto, Naito, \& Abe, 2016; Viswesvaran \& Ones 1999). These response biases are considered as one of the main sources of measurement error; therefore, they may harm the validity of the measurement (Podsakff, MacKenzie, Lee, \& Podsakoff, 2003).

One promising solution for dealing with response biases is to move away from the Likert measurement and instead present items in a comparative manner. In the two-alternative multidimensional forced-choice (2MFC) format, two paired statements are simultaneously presented as a questionnaire item. Respondents are asked to choose one of the two statements that best describes themselves. An example of 2MFC items is the pair of statements "Get stressed out easily" and "Don't talk a lot." Each statement in an item corresponds to different dimensions of psychological traits to be measured, such as emotional stability and extraversion in the preceding example. The $2 \mathrm{MFC}$ format should reduce the impact of uniform response biases because it makes it impossible to process uniformly elevated or decreased judgment across all items (Brown \& Maydeu-Olivares, 2018). In addition, there is evidence that the use of the forced-choice format can also reduce the influence of socially desirable responding and improve 
predictive validity (e.g., Christiansen, Burns, \& Montgomery, 2005; Jackson, Wroblewski, \& Ashton, 2000; Salgado \& Táuriz 2014).

However, when we code the $2 \mathrm{MFC}$ responses as binary scores, i.e., 1 for chosen and 0 for unchosen statements, the resultant variables become ipsative. Ipsative data suffer from statistical problems such as distorted validity (for more details, see e.g., Brown 2010; Brown \& Maydeu-Olivares, 2011; Hicks, 1970; Johnson, Wood, \& Blinkhorn, 1988).

A promising solution to the problem of ipsative data is to apply the framework of item response theory (IRT; Rasch, 1960; Lord, 1980) instead of summing the binarycoded scores. For this purpose, Brown and Maydeu-Olivares (2011) proposed a novel and general IRT model of forcedchoice format observations called the Thurstonian IRT model by extending Thurstone's Law of Comparative Judgment (Thurstone, 1927) to an IRT formulation. By incorporating the response process that is appropriate for forced-choice data, the Thurstonian IRT model succeeded in simultaneously estimating both item and respondent parameters and is free from the abovementioned problems of binary-coded ipsative data. To date, Thurstonian IRT is considered to be the only model that can be readily applied to data collected with existing multidimensional forcedchoice format questionnaires, with the additional objectives of estimating item parameters and correlations between the latent trait dimensions (Brown \& Maydeu-Olivares, 2018).

For ordinary Likert-scale measured personality data, another extension of the IRT model has recently attracted researchers' attention: the joint modeling of the item response and response time. The incorporation of the response time is particularly important because, nowadays, more and more questionnaires are conducted with computers instead of paper and pencils. In computer-based measurement, the response time information can be obtained with little additional cost along with the item responses. Various studies have discussed the utility of incorporating response time information in, e.g., classifying response behaviors, removing aberrant responses, and improving the estimation accuracy (e.g., Bertling and Weeks, 2018; Kong, Wise, \& Bhola, 2007; Wang, Xu, \& Shang, 2018; Wise \& Kong, 2005). The general validity of online measurement has been demonstrated for both personality characteristics and response times (e.g., Condon, 2018; Raz, Bar-Haim, Sadeh, \& Dan, 2014).

The D-diffusion IRT model (Tuerlinckx \& De Boeck, 2005) is a promising cognitive psychometric model (Batchelder, 1998; Vandekerckhove, 2014; Ranger et al., 2017) for the joint modeling of item responses and response times for personality questionnaire items. It combines two key modeling ideas: IRT models that have the virtue of disentangling respondent and item characteristics and cognitive process models that are grounded in cognitive theory to implement validated mechanisms for performing response tasks. Because IRT models were originally developed for ability measurement, many response-timeincorporated IRT models assume that the respondent is more skilled when they answer an item faster. However, the response time in personality measurement should have different characteristics. Namely, the response time tends to become longer when the relative position of a latent respondent parameter is close to the corresponding item parameters, regardless of the direction; this phenomenon has been called the distance-difficulty hypothesis (Kuncel, 1973; Ferrando \& Lorenzo-Seva, 2010). This relationship is analogous to a well-known psychophysical result that the uncertainty of responding to an stimulus is maximum when the stimulus is near the respondent's psychological threshold (Ferrando, 2006). To date, the distance-difficulty hypothesis has been a leading theory for explaining the response time of personality measurement items (Molenaar \& Bolsinova, 2017).

The D-diffusion IRT model, which will be mathematically introduced in the next section, implements this distance-difficulty relationship of personality measurement in a natural and quantitative manner. The model has a good parameter recovery property (Ranger, Kuhn, \& Szardenings, 2016) and has begun to be applied to empirical psychological data such as the measurement of extraversion (Molenaar, Tuerlinckx, \& Maas, 2015). Furthermore, by explicitly taking into account the cognitive process underlying the observed responses, the D-diffusion IRT model allows us to obtain psychologically meaningful parameter estimates such as the speed of information uptake and the amount of information used to make a decision. This helps us to quantify and empirically test psychological theories in an applied context (van Ravenzwaaij \& Oberauer, 2009).

However, so far, Thurstonian IRT and D-diffusion IRT models have taken two different pathways of model development. In fact, the authors are not aware of any cognitive psychometric models that can jointly model the item response and response time information of personality measurements collected in the 2MFC format. Consequently, the primary objective of the current study is to propose and examine such a model by integrating the Thurstonian IRT and Ddiffusion IRT models. A Bayesian modeling approach (Lee \& Wagenmakers, 2013) is adopted in the proposed method.

The remainder of this paper consists of five sections. In the "Existing models" section, we review the abovementioned existing models with more mathematical depth. Then, we introduce the proposed model, which is named the Thurstonian D-diffusion IRT model, in the following section. The "Simulation study" section describes a simulation study that was conducted to check parameter recovery. The "Real data application: Big-Five data" section provides the application of the proposed Thurstonian D-diffusion 
IRT model to real psychological data to demonstrate that it successfully combines the item response and response time information. Finally, a general discussion and possible directions for further research are provided in the "Discussion" section.

\section{Existing models}

\section{Diffusion model}

The following descriptions of the diffusion and D-diffusion IRT models are adapted and modified from Bunji and Okada (2019). The diffusion model (Ratcliff, 1978) is a model of the cognitive process that underlies a respondent's response in decision making tasks (for reviews, see Ratcliff \& McKoon, 2008; Voss, Nagler, \& Lerche, 2013). This model decomposes the observed item response and response time into several different psychologically meaningful parameters. One of the major advantages of the diffusion model is that it addresses the tradeoff between the response accuracy and the response speed thanks to this decomposition. This facilitates the quantification of individual differences in the cognitive processes. Figure 1 shows a schematic of the diffusion model. When an item is presented to the respondent, the cognitive information required to respond to the item accumulates over time from the starting point $(z)$. Once it reaches the upper $(a)$ or lower $(0)$ boundary, the respondent answers the item. The diffusion model was originally aimed at two-alternative forced-choice tasks, and the upper and lower boundaries correspond to each of these options. The increase in the amount of information in a unit time is assumed to follow a normal distribution with mean $v$ and variance $s^{2}$.

The diffusion model has four main parameters. $a$ represents the distance between the upper and lower boundaries. A larger value of $a$ means that a longer time is required to answer the item, which suggests that the respondent's choice is more deliberate and cautious in responding. $z$ is the

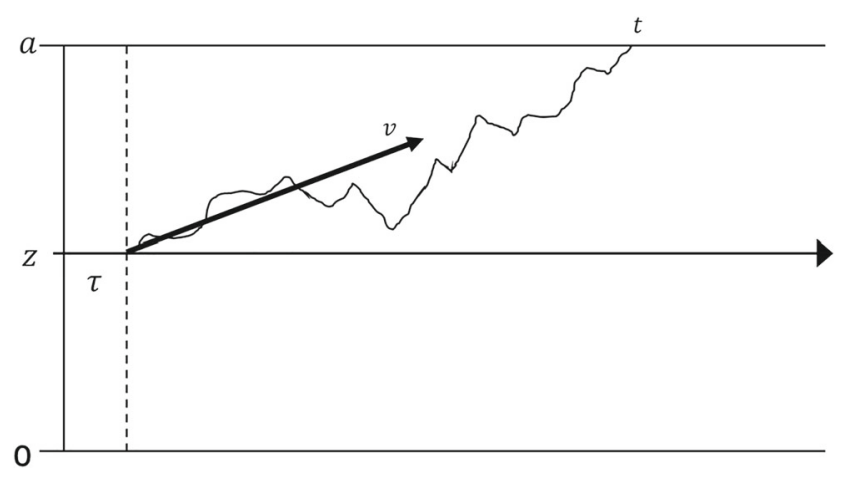

Fig. 1 Schematic of the diffusion model (figure adapted from Bunji \& Okada, 2019) starting point. When there is no initial bias towards either of the two boundaries, $z$ equals $a / 2$. $v$ represents the average slope of the information accumulation process. The process approaches the upper (resp. lower) bound when $v$ is positive (resp. negative). For identification of scale, the variance $s^{2}$ is commonly fixed as $s^{2}=1 . \tau$ represents the nonresponse time, i.e., the duration of nondecisional processes that may comprise basic encoding processes.

\section{D-diffusion IRT model}

Tuerlinckx and De Boeck (2005) extended the diffusion model to the IRT framework, followed by its generalization by van der Maas, Molenaar, Maris, Kievit, and Borsboom (2011). Broadly speaking, there exist two typical occasions for which IRT models are used for psychological measurement: personality measurement, e.g., for the Big-Five traits, and ability measurement, e.g., for college entrance examinations. Corresponding to these, two types of diffusion IRT models with different functional forms have been developed: the D- and Q-diffusion IRT models for the former and latter, respectively (Tuerlinckx \& De Boeck, 2005; van der Maas et al., 2011). Because our interest is in personality measurement, we consider the D-diffusion IRT model in this paper.

Let $x_{i j}$ be the binary observed item response that takes values of 1 when respondent $i$ chooses the first option for item $j$ (or the accumulation reaches the upper bound) and 0 when the second option is chosen (the accumulation reaches the lower bound). Moreover, let $t_{i j}$ be the corresponding response time. When $x_{i j}$ and $t_{i j}$ are generated by the diffusion model, the joint distribution of the item response $\left(x_{i j}\right)$ and response time $\left(t_{i j}\right)$ can be expressed as

$$
\begin{aligned}
f\left(x_{i j}, t_{i j}\right) & =\frac{\pi s^{2}}{a_{i j}^{2}} \exp \left(\frac{\left(a_{i j} x_{i j}-z_{i j}\right) v_{i j}}{s^{2}}-\frac{v_{i j}^{2}}{2 s^{2}}\left(t_{i j}-\tau_{j}\right)\right) \\
& \times \sum_{m=1}^{\infty} m \sin \left(\frac{\pi m\left(a_{i j} x_{i j}-2 z_{i j} x_{i j}+z_{i j}\right)}{a_{i j}}\right) \\
& \times \exp \left(-\frac{1}{2} \frac{\pi^{2} s^{2} m^{2}}{a_{i j}^{2}}\left(t_{i j}-\tau_{j}\right)\right) .
\end{aligned}
$$

In typical personality measurement, it would be reasonable to assume that there is no response bias at the starting point. Under the assumption that $z_{i j}$ is set to $a_{i j} / 2$, which indicates no initial response bias, the probability that respondent $i$ chooses the first option for the $j$-th item is given as

$P\left(x_{i j}=1\right)=\frac{\exp \left(-2 z_{i j} v_{i j}\right)-1}{\exp \left(-2 a_{i j} v_{i j}\right)-1}=\frac{\exp \left(a_{i j} v_{i j}\right)}{1+\exp \left(a_{i j} v_{i j}\right)}$,

which is a form of the logistic curve. 
In the D-diffusion IRT model, the diffusion model parameters are decomposed into the respondent and item IRT parameters. Specifically, the diffusion model parameters are expressed as

$$
\left\{\begin{array}{lll}
a_{i j}=\frac{\gamma_{i}}{\xi_{j}} & \text { with } & \gamma_{i}, \xi_{j} \in \mathbb{R}_{>0} \\
v_{i j}=\theta_{i}-b_{j} & \text { with } & \theta_{i}, b_{j} \in \mathbb{R} .
\end{array}\right.
$$

Notably, $v_{i j}$ is expressed as the difference between the item difficulty and the respondent's latent trait, hence the name D-diffusion IRT. Further, the distance between the upper and lower boundaries, $a_{i j}$, is given as the ratio of the respondent properties (e.g., attentiveness and deliberateness) and the item properties (e.g., complexity and length). Such a separation between item and respondent parameters is a key characteristic of IRT models. This is in contrast to the original diffusion model, in which the separation between the two was not explicitly taken care of.

As a result, Eq. 2 is rewritten as

$$
P\left(x_{i j}=1 \mid \theta_{i}, \gamma_{i}\right)=\frac{\exp \left(\frac{\gamma_{i}}{\xi_{j}}\left(\theta_{i}-b_{j}\right)\right)}{1+\exp \left(\frac{\gamma_{i}}{\xi_{j}}\left(\theta_{i}-b_{j}\right)\right)},
$$

which is the form of the two-parameter logistic IRT model except that the discrimination parameter $\gamma_{i} / \xi_{j}$ depends on both the respondent and item. $\tau_{j}$ retains the same meaning as in the original diffusion model, and we considered $\tau_{j}$ as an item parameter in this study. Owing to a property of the diffusion model, the expected response time is the longest when $v_{i j}=\theta_{i}-b_{j}=0$. This means that, when the respondent's latent trait is closer to the item difficulty, the expected response time becomes longer, which is the expectation of the distance-difficulty hypothesis. In this way, the D-diffusion IRT model generates important predictions for personality measurement.

\section{Thurstonian IRT model}

As previously stated, the Thurstonian IRT model (Brown \& Maydeu-Olivares, 2011) extends Thurstone's Law of Comparative Judgment (Thurstone, 1927) to the IRT framework. Let $j_{1}$ and $j_{2}$ be paired statements presented as the $j$-th item, and let $x_{i j}$ be the observed binary response of respondent $i$ that takes values of 1 when $j_{1}$ is chosen and 0 otherwise. In addition, let $u_{i j_{1}}$ and $u_{i j_{2}}$ be the latent utilities of respondent $i$ for $j_{1}$ and $j_{2}$, respectively. In the Thurstonian IRT model, the observed response is modeled to be determined by the difference between $u_{i j_{1}}$ and $u_{i j_{2}}$ of that respondent; that is,

$x_{i j}=\left\{\begin{array}{l}1 \text { if } u_{i j_{1}} \geq u_{i j_{2}} \\ 0 \text { if } u_{i j_{1}}<u_{i j_{2}} .\end{array}\right.$

This means that the observed item response depends on the sign of the difference between the latent utilities: $x_{i j}^{*}=$ $u_{i j_{1}}-u_{i j_{2}}$. In the 2MFC measurement of personality, each statement is designed to measure different trait dimensions; for instance, the first statement measures emotional stability, and the second statement measures extraversion. Let $m_{j_{1}}$ and $m_{j_{2}}$ denote the latent trait dimensions that are measured by $j_{1}$ and $j_{2}$, respectively, where $M$ is the total number of latent traits to be measured in the questionnaire, $1 \leq m_{j_{1}} \leq$ $M, 1 \leq m_{j_{2}} \leq M$, and $m_{j_{1}} \neq m_{j_{2}}$.

Here, $u_{i j_{1}}$ and $u_{i j_{2}}$ can be written as the Thurstonian factor model:

$u_{i j_{1}}=\mu_{j_{1}}+\lambda_{j_{1}} \eta_{i m_{j_{1}}}+\epsilon_{i j_{1}}$,

$u_{i j_{2}}=\mu_{j_{2}}+\lambda_{j_{2}} \eta_{i m_{j_{2}}}+\epsilon_{i j_{2}}$,

where $\mu_{j_{k}}$ represents the mean of the latent utility $u_{i j_{k}}, \lambda_{j_{k}}$ represents a factor loading, $\eta_{i m_{j_{k}}}$ represents the latent trait of respondent $i$, and $\epsilon_{i j_{k}}$ represents the residual or unique factor. Both $\boldsymbol{\eta}_{i}$ and $\boldsymbol{\epsilon}_{i}$ are assumed to be (multivariate) normally distributed.

In order to facilitate understanding of the notation, let us consider the example in Table 3 , in which $M=5$ latent traits are measured using $J=25$ items (i.e., pairs of statements). In item 2 , the first and second statements measure agreeableness and intellect/imagination, respectively. They correspond to the third and fifth traits (as indicated in the footnote of the table). Therefore, in this case, $m_{2_{1}}=3$, and $m_{2_{2}}=5$. As a result, Eq. 6 for item 2 in Table 3 is given as

$u_{i 2_{1}}=\mu_{2_{1}}+\lambda_{2_{1}} \eta_{i 3}+\epsilon_{i 2_{1}}$,

$u_{i 2_{2}}=\mu_{2_{2}}+\lambda_{2_{2}} \eta_{i 5}+\epsilon_{i 2_{2}}$.

Brown and Maydeu-Olivares (2011) proposed their Thurstonian IRT model by reparameterizing the above second-order Thurstonian factor model. Specifically, its item response function is given by

$P\left(x_{i j}=1 \mid \boldsymbol{\eta}_{i}\right)=\boldsymbol{\Phi}\left(\frac{\lambda_{j_{1}} \eta_{i m_{j_{1}}}-\lambda_{j_{2}} \eta_{i m_{j_{2}}}-\delta_{j}}{\sqrt{\psi_{j_{1}}^{2}+\psi_{j_{2}}^{2}}}\right)$,

where $\boldsymbol{\Phi}(\cdot)$ denotes the cumulative distribution function of the standard normal distribution, $\delta_{j}$ represents the difference between the means of the latent utility $\mu_{j_{k}}$, and $\psi_{j_{k}}^{2}$ represents the variance of the unique factor $\epsilon_{i j_{k}}$. By using the reparameterization

$\alpha_{j}=\frac{\delta_{j}}{\sqrt{\psi_{j_{1}}^{2}+\psi_{j_{2}}^{2}}}, \quad \beta_{j_{1}}=\frac{\lambda_{j_{1}}}{\sqrt{\psi_{j_{1}}^{2}+\psi_{j_{2}}^{2}}}, \quad \beta_{j_{2}}=\frac{\lambda_{j_{2}}}{\sqrt{\psi_{j_{1}}^{2}+\psi_{j_{2}}^{2}}}$, 
Eq. 8 can be rewritten as

$P\left(x_{i j}=1 \mid \eta_{i}\right)=\boldsymbol{\Phi}\left(\beta_{j_{1}} \eta_{i m_{j_{1}}}-\beta_{j_{2}} \eta_{i m_{j_{2}}}-\alpha_{j}\right)$,

which corresponds to the form of the two-dimensional normal ogive IRT model. When an item consists of three or more statements, i.e., when we need to include comparisons between more than two statements simultaneously, $\alpha_{j}$ and $\beta_{j_{k}}$ become mathematically dependent parameters. However, when an item consists of only two statements, which is the case considered in this paper, we can simply estimate them as free parameters (Brown \& MaydeuOlivares, 2011).

\section{Thurstonian D-diffusion IRT model}

Obviously, Eqs. 4 and 10 have similar functional forms, as a logistic function can be considered as an approximation to the normal ogive function. In addition, both respondent parameters, $\theta_{i}$ in the diffusion IRT model and $\boldsymbol{\eta}_{i}$ in the Thurstonian IRT model, have a similar empirical meaning and the same distributional form. Therefore, when combining these two models, we can simply replace $\left(\theta_{i}-\right.$ $\left.b_{j}\right)$ in Eq. 4 with $\left(\beta_{j_{1}} \eta_{i m_{j_{1}}}-\beta_{j_{2}} \eta_{i m_{j_{2}}}-\alpha_{j}\right)$ in Eq. 10. However, these two models have different forms for the discrimination parameter. In the diffusion IRT model, the discrimination parameter is the quotient of the respondent parameter $\gamma_{i}$ divided by the item parameter $\xi_{j}$. Therefore, in the diffusion IRT model, the discriminability depends on both item factors such as the statement length and time limit and respondent factors such as the response caution and temper (van der Maas et al., 2011). On the other hand, the slope and intercept parameters in the original Thurstonian IRT model are statement and item parameters, respectively. Therefore, the discriminability in the Thurstonian IRT model is not affected by respondent factors.

On the basis of the above fact, in introducing the proposed model, we start from the Thurstonian IRT model and extend it so that the slope and intercept parameters in Eq. 10 depend on both the item and respondent factors. The Thurstonian IRT model maintains its identifiability even if we simply multiply $\left(\beta_{j_{1}} \eta_{i m_{j_{1}}}-\beta_{j_{2}} \eta_{i m_{j_{2}}}-\alpha_{j}\right)$ in Eq. 10 by $\left(\xi_{j} / \gamma_{i}\right)$ because $\beta_{j_{k}}$ is a statement parameter; that is, each statement independently has its own $\beta_{j_{k}}$. This parameter may be interpreted as the discriminability of the specified statement. On the other hand, $\xi_{j}$ is an item parameter that is specific to the pair of statements. This means that $\xi_{j}$ represents an effect that is inherent to the paired statements that are simultaneously presented to respondents.

The above arguments lead us to introduce the proposed Thurstonian D-diffusion IRT model, which we develop as an extension of the D-diffusion IRT model. Specifically, in the proposed model, the joint distribution of the item response and response time is given as Eq. 1. However, in its marginalized IRT-type form of Eq. 2, we reparameterize the two parameters $a_{i j}$ and $v_{i j}$ in the same manner as in the case of Thurstonian IRT as

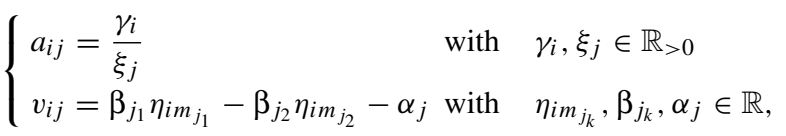

instead of Eq. 4.

Then, the joint likelihood of the item response and response time in the proposed model is given as

$$
\begin{aligned}
f\left(x_{i j}, t_{i j}\right)= & \frac{\pi s^{2} \xi_{j}^{2}}{\gamma_{i}^{2}} \exp \left(\frac{\gamma_{i}\left(\beta_{j_{1}} \eta_{i m_{j_{1}}}-\beta_{j_{2}} \eta_{i m_{j_{2}}}-\alpha_{j}\right)\left(2 x_{i j}-1\right)}{2 s^{2} \xi_{j}}\right. \\
& \left.-\frac{\left(\beta_{j_{1}} \eta_{i m_{j_{1}}}-\beta_{j_{2}} \eta_{i m_{j_{2}}}-\alpha_{j}\right)^{2}\left(t_{i j}-\tau_{j}\right)}{2 s^{2}}\right) \\
& \times \sum_{m=1}^{\infty} m \sin \left(\frac{\pi m}{2}\right) \exp \left(-\frac{1}{2} \frac{\pi^{2} s^{2} m^{2} \xi_{j}^{2}}{\gamma_{i}^{2}}\left(t_{i j}-\tau_{j}\right)\right) .
\end{aligned}
$$

In practice, Eq. 12 cannot be directly calculated because it involves the sum of an infinite series. Therefore, Navarro and Fuss's (2009) approximation is commonly used for its evaluation. This approach is also employed in stan, which is the software we used in this study.

From Eq. 12, the probability of choosing the first statement, which is a counterpart of both Eqs. 4 and 10, is then given as

$$
P\left(x_{i j}=1 \mid \boldsymbol{\eta}_{i}\right)=\frac{\exp \left(\frac{\gamma_{i}}{\xi_{j}}\left(\beta_{j_{1}} \eta_{i m_{j_{1}}}-\beta_{j_{2}} \eta_{i m_{j_{2}}}-\alpha_{j}\right)\right)}{1+\exp \left(\frac{\gamma_{i}}{\xi_{j}}\left(\beta_{j_{1}} \eta_{i m_{j_{1}}}-\beta_{j_{2}} \eta_{i m_{j_{2}}}-\alpha_{j}\right)\right)} .
$$

In this study, we assume that the researcher already knows the keyed direction of each statement because this is natural in personality measurement using existing scales. Therefore, the sign of $\beta_{j_{k}}$ is known. We first estimate $\beta_{j_{k}}$ under the restriction of positivity. Then, we manually invert the sign of the obtained negatively keyed item parameter estimates in order to facilitate their interpretations.

Brown and Maydeu-Olivares (2011) recommends designing a forced-choice questionnaire by preparing both types of items that are keyed in the same direction (e.g., both statements are positively keyed) and those in the opposite direction (one statement is positively keyed and the other negatively keyed) in order to ensure accurate measurement of traits. We recommend the same in the proposed approach.

When the number of latent traits measured in a $2 \mathrm{MFC}$ questionnaire is two $(M=2)$, the proposed model, 
as well as the Thurstonian IRT model, suffers from the identification problem. This can be readily understood because in this case, all statements have nonzero factor loadings to all traits, which is essentially the case of an exploratory factor analysis. Brown and Maydeu-Olivares $(2011,2012)$ dealt with this problem by fixing the factor loadings of the first two statements to their true values in the simulation study. Obviously, this approach is not realistic in practice because a researcher never knows the true factor loading parameter values. Therefore, in this paper, we only consider the case when $M>2$ and recommend applying the proposed model for measuring more than two traits.

The Bayesian model is completed by placing prior distributions over the parameters. We use the following priors throughout this paper:

$$
\begin{aligned}
& \xi_{j} \sim t_{[0, \infty)}(4,0,2.5), \quad \gamma_{i} \sim \operatorname{LN}(0,1), \\
& \beta_{j_{k}} \sim t_{[0, \infty)}(4,0,2.5), \boldsymbol{\eta}_{i} \sim M V N(\mathbf{0}, \boldsymbol{\Sigma}), \\
& \alpha_{j} \sim N(0,2.5), \quad \tau_{j} \sim U\left(0, \min (\mathrm{RT})_{j}\right) \text {, } \\
& \boldsymbol{\Sigma} \sim L K J \operatorname{Corr}(1) \text {, }
\end{aligned}
$$

where $\min (\mathrm{RT})_{j}$ represents the minimum observed response time for each item $j$. Our basic principle in specifying the priors here is to place standardized distributions for the respondent parameters and weakly informative priors that give very low probabilities to very implausible parameter values for item parameters. The priors for the respondent parameters are standardized for identification reasons. Hence, the variance of each latent trait is fixed to 1 . This means that the covariance matrix $\boldsymbol{\Sigma}$ reduces to a correlation matrix. Hence, we call $\boldsymbol{\Sigma}$ as the correlation matrix hereafter and denote its elements by $\rho_{\mathrm{mm}^{\prime}}$. Moreover, $\gamma_{i}$ follows the standard lognormal distribution, and the variance of the drift rate $\left(s^{2}\right)$ is set to be 1 . The LKJ correlation distribution (Lewandowski, Kurowicka, \& Joe, 2009) for the correlation matrix here corresponds to a uniform prior over all possible $M \times M$ correlation matrices (Stan Development Team, 2017). For the priors of the item parameters, we adopted the half $-t$ distribution for $\xi_{j}$ and $\beta_{j_{k}}$ as weakly informative priors, which are recommended by Gelman (2006) and Stan Development Team (2017).

The parameters of the proposed Thurstonian D-diffusion IRT model can be estimated using the Markov Chain Monte Carlo (MCMC) algorithm. For all of the estimation results presented below, we used R 3.5.0 and stan 2.17.3 on a Windows 10 PC. Three MCMC chains were run for each dataset. The number of MCMC iterations per chain was 10,000 , half of which were discarded as warm-up. The data and computer codes (Stan and R) that we used for real data application are available from the Open Science Framework website (https://osf.io/jswqg/). We used the posterior means of the parameters as their point estimates.

\section{Simulation study}

We conducted a simulation study to check the parameter recovery properties of the proposed model. We generated simulation data from the Thurstonian D-diffusion IRT model with some narrower distributions described below and estimated the parameter values from the generated data with the conventional priors described in the previous section.

We considered the following five scenarios in this simulation:

- The first scenario manipulated the two crossed factors-the number of trait dimensions $M=(3,4)$ and the number of items that consist of the same pairs of dimensions $J_{\text {pair }}=(3,5,7)$-to examine their effects on parameter recovery. Note that the total number of items $J$ is related to these factors by

$J=J_{\text {pair }} \times \frac{M(M-1)}{2}$.

The trait dimensions were independent of one another in this scenario and the second scenario.

- The second scenario examined the influence of the number of dimensions $M$ when the questionnaire length was fixed. For this purpose, we manipulated the number of dimensions $M=(3,4)$ under conditions where the total number of items $J=(12,24,36)$.

- The third and fourth scenarios examined if the proposed model can properly recover the parameter values even when the dimensions are correlated. The factor examined in the third scenario was the correlations between the dimensions, which correspond to the off-diagonal elements of $\boldsymbol{\Sigma}$. All of the off-diagonal elements were set to have the same values, which we denote by $\rho_{\text {pair }}$. Its three manipulated conditions were $\rho_{\text {pair }}=(-0.3,0.3,0.5)$. In this scenario, we set $M=3$ and $J_{\text {pair }}=5$.

- The fourth scenario considered more realistic conditions and attempted to emulate the Big-Five factor structure. Specifically, the correlation matrix $\boldsymbol{\Sigma}$ was specified on the basis of Brown and Maydeu-Olivares (2011) as

$$
\boldsymbol{\Sigma}=\left(\begin{array}{cccccc}
1 & & & & \\
-.21 & 1 & & & \\
0 & .40 & 1 & & \\
-.25 & 0 & 0 & 1 & \\
-.53 & .27 & 0 & .24 & 1
\end{array}\right)
$$


Table 1 Mean RMSEs of the parameter estimates in the simulation study

\begin{tabular}{|c|c|c|c|c|c|c|c|c|c|c|c|}
\hline \multirow[b]{2}{*}{$M$} & \multirow[b]{2}{*}{$J_{\text {pair }}$} & \multirow[b]{2}{*}{$J$} & \multirow[b]{2}{*}{$\rho_{\text {pair }}$} & \multirow[b]{2}{*}{$\xi_{j} \sim$} & \multicolumn{7}{|c|}{ Mean RMSE } \\
\hline & & & & & $\xi$ & $\beta$ & $\alpha$ & $\tau$ & $\log (\gamma)$ & $\eta$ & $\rho$ \\
\hline 3 & 3 & 9 & 0 & $\mathrm{U}(0.3,0.7)$ & 0.034 & 0.126 & 0.106 & 0.001 & 0.166 & 0.564 & 0.059 \\
\hline 3 & 5 & 15 & 0 & $\mathrm{U}(0.3,0.7)$ & 0.035 & 0.105 & 0.108 & 0.001 & 0.136 & 0.487 & 0.052 \\
\hline 3 & 7 & 21 & 0 & $\mathrm{U}(0.3,0.7)$ & 0.043 & 0.107 & 0.095 & 0.002 & 0.129 & 0.433 & 0.037 \\
\hline 4 & 3 & 18 & 0 & $\mathrm{U}(0.3,0.7)$ & 0.042 & 0.102 & 0.106 & 0.001 & 0.136 & 0.494 & 0.046 \\
\hline 4 & 5 & 30 & 0 & $\mathrm{U}(0.3,0.7)$ & 0.056 & 0.112 & 0.102 & 0.001 & 0.137 & 0.422 & 0.040 \\
\hline 4 & 7 & 42 & 0 & $\mathrm{U}(0.3,0.7)$ & 0.065 & 0.102 & 0.107 & 0.002 & 0.139 & 0.390 & 0.038 \\
\hline 3 & 4 & 12 & 0 & $\mathrm{U}(0.3,0.7)$ & 0.026 & 0.112 & 0.106 & 0.001 & 0.142 & 0.513 & 0.052 \\
\hline 4 & 2 & 12 & 0 & $\mathrm{U}(0.3,0.7)$ & 0.044 & 0.126 & 0.113 & 0.001 & 0.160 & 0.558 & 0.054 \\
\hline 3 & 8 & 24 & 0 & $\mathrm{U}(0.3,0.7)$ & 0.053 & 0.107 & 0.100 & 0.001 & 0.137 & 0.417 & 0.034 \\
\hline 4 & 4 & 24 & 0 & $\mathrm{U}(0.3,0.7)$ & 0.054 & 0.110 & 0.143 & 0.010 & 0.178 & 0.478 & 0.043 \\
\hline 3 & 12 & 36 & 0 & $\mathrm{U}(0.3,0.7)$ & 0.072 & 0.108 & 0.107 & 0.001 & 0.153 & 0.366 & 0.036 \\
\hline 4 & 6 & 36 & 0 & $\mathrm{U}(0.3,0.7)$ & 0.075 & 0.107 & 0.142 & 0.011 & 0.193 & 0.429 & 0.036 \\
\hline 3 & 5 & 15 & -0.3 & $\mathrm{U}(0.3,0.7)$ & 0.041 & 0.115 & 0.108 & 0.001 & 0.144 & 0.475 & 0.054 \\
\hline 3 & 5 & 15 & 0.3 & $\mathrm{U}(0.3,0.7)$ & 0.040 & 0.113 & 0.106 & 0.001 & 0.143 & 0.478 & 0.056 \\
\hline 3 & 5 & 15 & 0.5 & $\mathrm{U}(0.3,0.7)$ & 0.033 & 0.113 & 0.098 & 0.001 & 0.132 & 0.474 & 0.047 \\
\hline 5 & 3 & 30 & $B F$ & $\mathrm{U}(0.3,0.7)$ & 0.050 & 0.111 & 0.113 & 0.001 & 0.128 & 0.437 & 0.038 \\
\hline 5 & 5 & 50 & $B F$ & $\mathrm{U}(0.3,0.7)$ & 0.099 & 0.116 & 0.108 & 0.001 & 0.189 & 0.378 & 0.035 \\
\hline 5 & 7 & 70 & $B F$ & $\mathrm{U}(0.3,0.7)$ & 0.138 & 0.129 & 0.105 & 0.001 & 0.246 & 0.343 & 0.035 \\
\hline 3 & 5 & 15 & 0 & $\mathrm{U}(0.2,0.3)$ & 0.019 & 0.084 & 0.096 & 0.004 & 0.138 & 0.355 & 0.035 \\
\hline 3 & 5 & 15 & 0 & $\mathrm{U}(0.4,0.5)$ & 0.035 & 0.106 & 0.106 & 0.002 & 0.142 & 0.473 & 0.051 \\
\hline 3 & 5 & 15 & 0 & $\mathrm{U}(0.6,0.7)$ & 0.045 & 0.113 & 0.127 & 0.001 & 0.137 & 0.546 & 0.053 \\
\hline
\end{tabular}

Note: $B F=$ correlation between traits based onBrown and Maydeu-Olivares (2011)

We denote this as $\rho_{\text {pair }}=B F$ for simplicity. In this scenario, $M=5$, and $J_{\text {pair }}$ was manipulated for three conditions, which were $J_{\text {pair }}=(3,5,7)$.

- The fifth scenario examined the influence of the complexity (cognitive workload) of the items. In this scenario, we manipulated the true distribution of $\xi_{j}$ to be either $U(0.2,0.3), U(0.4,0.5)$, or $U(0.6,0.7)$. Here, we fixed $M=3, J_{\text {pair }}=5$ and assumed no correlations among traits.

This results in a total of 21 conditions. The number of respondents was kept constant at $I=300$ for all conditions. In each condition, the cycle of random data generation and parameter estimation was replicated 30 times.

\section{Data generation}

The distributions used to generate random data from the proposed model were as follows: $\gamma_{i} \sim L N(0,1), \beta_{j_{k}} \sim$ $U(0.5,1.5), \eta_{i} \sim N(\mathbf{0}, \boldsymbol{\Sigma}), \alpha_{j} \sim U(-1.5,1.5)$, and $\tau_{j} \sim U(0.2,1)$. With regard to $\xi_{j}$, we drew its samples from $U(0.3,0.7)$ in the first to fourth scenarios. These distributions are chosen on the basis of our preliminary analysis, Brown and Maydeu-Olivares (2011) and Bunji and
Okada (2019). Note that the off-diagonal elements of the correlation matrix $\boldsymbol{\Sigma}$ were treated as separate parameters and estimated as such even when the true correlations had the same values. Moreover, for a practical reason, ${ }^{1}$ observations with a response time greater than $120 \mathrm{~s}$ were listwise deleted. We considered this manipulation to be acceptable because an observation with such a large response time may be considered as an irregular response.

\section{Results}

Tables 1 and 2 summarize the means of the root mean square errors (RMSEs) and the means of the biases for each condition, respectively. The RMSE for the parameter $\beta$, for example, is calculated by

$\operatorname{RMSE}_{\beta}=\sqrt{\frac{1}{J} \frac{1}{2} \sum_{j=1}^{J} \sum_{k=1}^{2}\left(\hat{\beta}_{j_{k}}-\beta_{j_{k}}\right)^{2}}$,

\footnotetext{
${ }^{1}$ When estimated with Stan, the built-in wiener_lpdf function sometimes produces $\log (0)$ and returns an error. This error occurs more often when the response time is longer.
} 
where $\beta_{j_{k}}$ is the realized value that is generated from $U(0.5,1.5)$ in each iteration and $\hat{\beta}_{j_{k}}$ is its expected a posteriori estimate. Similarly, the bias for the parameter $\eta$, for example, is calculated by

$\operatorname{bias}_{\eta}=\frac{1}{I} \frac{1}{M} \sum_{i=1}^{I} \sum_{m=1}^{M}\left(\hat{\eta}_{i m}-\eta_{i m}\right)$.

Since $\gamma_{i}$ is lognormally distributed, the RMSEs and biases of the log-transformed estimates for $\gamma$ are presented. The RMSEs for both $\beta$ and $\rho$ are acceptably small when $M=3$ and $J_{\text {pair }}=3$, even when $J$ is as small as 9. These results suggest the proposed model is capable of appropriately estimating the trait loadings $\beta_{j_{k}}$ and the correlations between traits $\rho_{m m^{\prime}}$.

In the second scenario, the RMSEs for $\eta$ become larger when the number of traits increases. Therefore, this suggests that a smaller number of traits would provide greater accuracy in the estimation of $\eta_{i m}$. Further, since the RMSE decreases as $J$ increases, it would be desirable to have many items in the questionnaire if possible.

The RMSEs for $\eta$ might look rather large for all conditions. However, these values are actually comparable in scale with the values reported by Stone (1992) when the number of items for each factor is larger than 10 (for example, the number of items for each factor is 10 when $M=3$ and $J_{\text {pair }}=5$ ).

A similar estimation accuracy is obtained when nonzero correlations exist between traits, which corresponds to the third and fourth scenarios. In addition, the trait correlations are successfully recovered for all conditions.

From the results of the fifth scenario, we can see that the estimation accuracy of $\eta_{i m}$ increases as the true distribution of $\xi_{j}$ shrinks. In other words, given the same number of items, the estimation accuracy tends to be better as the items require more cognitive workload. This relationship is consistent with the specifications of the diffusion IRT model, in which the item discrimination is equal to the boundary parameter, because a smaller $\xi_{j}$ leads to higher discrimination.

The means of the biases for $\xi$ and $\log (\gamma)$ are positive for almost all conditions. They become larger according to the increase in the total number of items $J$. In the same manner, the mean RMSEs for these parameters also become larger in accordance with the increase in $J$. The reason for these results can be understood from the form of the parameter constraints, as described below. $\xi_{j}$ and $\gamma_{i}$ originally have a mutual sign indeterminacy because they are given in the

Table 2 Mean biases of the parameter estimates in the simulation study

\begin{tabular}{|c|c|c|c|c|c|c|c|c|c|c|c|}
\hline \multirow[b]{2}{*}{$M$} & \multirow[b]{2}{*}{$J_{\text {pair }}$} & \multirow[b]{2}{*}{$J$} & \multirow[b]{2}{*}{$\rho_{\text {pair }}$} & \multirow[b]{2}{*}{$\xi_{j} \sim$} & \multicolumn{7}{|c|}{ Mean bias } \\
\hline & & & & & $\xi$ & $\beta$ & $\alpha$ & $\tau$ & $\log (\gamma)$ & $\eta$ & $\rho$ \\
\hline 3 & 3 & 9 & 0 & $\mathrm{U}(0.3,0.7)$ & 0.010 & 0.017 & 0.012 & 0.000 & 0.035 & 0.001 & 0.000 \\
\hline 3 & 5 & 15 & 0 & $\mathrm{U}(0.3,0.7)$ & 0.023 & 0.011 & 0.001 & 0.000 & 0.051 & -0.002 & 0.003 \\
\hline 3 & 7 & 21 & 0 & $\mathrm{U}(0.3,0.7)$ & 0.035 & 0.012 & 0.002 & 0.000 & 0.073 & 0.003 & -0.002 \\
\hline 4 & 3 & 18 & 0 & $\mathrm{U}(0.3,0.7)$ & 0.032 & 0.011 & 0.005 & 0.000 & 0.067 & 0.000 & 0.002 \\
\hline 4 & 5 & 30 & 0 & $\mathrm{U}(0.3,0.7)$ & 0.049 & 0.013 & 0.004 & 0.000 & 0.101 & 0.001 & -0.003 \\
\hline 4 & 7 & 42 & 0 & $\mathrm{U}(0.3,0.7)$ & 0.055 & 0.011 & 0.009 & 0.000 & 0.106 & -0.006 & 0.000 \\
\hline 3 & 4 & 12 & 0 & $\mathrm{U}(0.3,0.7)$ & 0.014 & 0.013 & -0.005 & 0.000 & 0.038 & -0.006 & -0.006 \\
\hline 4 & 2 & 12 & 0 & $\mathrm{U}(0.3,0.7)$ & 0.029 & 0.016 & 0.014 & 0.000 & 0.072 & 0.009 & 0.001 \\
\hline 3 & 8 & 24 & 0 & $\mathrm{U}(0.3,0.7)$ & 0.044 & 0.012 & 0.011 & 0.000 & 0.089 & 0.002 & -0.002 \\
\hline 4 & 4 & 24 & 0 & $\mathrm{U}(0.3,0.7)$ & 0.044 & 0.015 & 0.007 & 0.002 & 0.087 & 0.004 & -0.007 \\
\hline 3 & 12 & 36 & 0 & $\mathrm{U}(0.3,0.7)$ & 0.066 & 0.012 & 0.000 & 0.000 & 0.126 & 0.009 & 0.006 \\
\hline 4 & 6 & 36 & 0 & $\mathrm{U}(0.3,0.7)$ & 0.066 & 0.015 & -0.014 & 0.003 & 0.129 & 0.000 & 0.001 \\
\hline 3 & 5 & 15 & -0.3 & $\mathrm{U}(0.3,0.7)$ & 0.027 & 0.014 & -0.020 & 0.000 & 0.060 & -0.002 & -0.008 \\
\hline 3 & 5 & 15 & 0.3 & $\mathrm{U}(0.3,0.7)$ & 0.025 & 0.013 & 0.005 & 0.000 & 0.056 & 0.000 & 0.007 \\
\hline 3 & 5 & 15 & 0.5 & $\mathrm{U}(0.3,0.7)$ & 0.019 & 0.014 & 0.005 & 0.000 & 0.047 & -0.003 & -0.008 \\
\hline 5 & 3 & 30 & $B F$ & $\mathrm{U}(0.3,0.7)$ & 0.044 & 0.012 & 0.008 & 0.000 & 0.090 & 0.008 & -0.031 \\
\hline 5 & 5 & 50 & $B F$ & $\mathrm{U}(0.3,0.7)$ & 0.094 & 0.014 & -0.003 & 0.000 & 0.177 & -0.005 & -0.028 \\
\hline 5 & 7 & 70 & $B F$ & $\mathrm{U}(0.3,0.7)$ & 0.133 & 0.017 & 0.010 & 0.000 & 0.239 & 0.002 & -0.034 \\
\hline 3 & 5 & 15 & 0 & $\mathrm{U}(0.2,0.3)$ & 0.011 & 0.007 & 0.005 & 0.000 & 0.047 & -0.002 & -0.002 \\
\hline 3 & 5 & 15 & 0 & $\mathrm{U}(0.4,0.5)$ & 0.023 & 0.012 & 0.005 & 0.000 & 0.059 & 0.000 & -0.001 \\
\hline 3 & 5 & 15 & 0 & $\mathrm{U}(0.6,0.7)$ & 0.024 & 0.013 & -0.001 & 0.000 & 0.045 & 0.009 & 0.002 \\
\hline
\end{tabular}

Note: $B F=$ correlation between traits based on Brown and Maydeu-Olivares (2011) 
form of a quotient. To avoid this indeterminacy, a parameter constraint needs to be introduced. In this study, we chose the prior distribution for $\gamma_{i}$ to be the standard lognormal distribution. The effect of the priors tends to be reduced, corresponding to the increase in the data size, and this may explain the result that larger estimates for both $\xi_{j}$ and $\gamma_{i}$ tend to be obtained for larger $J$. However, we note that these biases and RMSEs are still practically sufficiently small to be acceptable. Moreover, they do not affect the relative order of the respondents or items effects, which should be more practically important. Furthermore, the biases for the other parameters are nearly zero and neither systematically positive nor negative.

\section{Sensitivity analysis}

In order to check that our prior specification does not actually have a strong influence on parameter estimation, we conducted a sensitivity analysis. In this sensitivity analysis, we considered the second scenario and changed the priors for the item parameters to be more diffuse ones. Specifically, the priors for the parameters $\xi_{j}, \beta_{j_{k}}$, and $\alpha_{j}$ were set as a diffuse $N(0,100)$ prior, and parameter recovery was comparatively checked. The other conditions of the simulation were the same as before. The results suggest that the change in the priors has only a minor influence on the parameter estimates (see Table A1 in the Online Supplementary Material). Considering the estimation efficiency, we think that it is practically adequate to employ the prior specification of Eq. 14, provided that the sample size is not very small.

To summarize the simulation results, the proposed Thurstonian D-diffusion IRT model is found to have sufficient parameter recovery properties.

\section{Real data application: Big-Five data}

In this section, we demonstrate the application of the proposed model to real $2 \mathrm{MFC}$ format personality measurement

Table 3 List of items (pairs of statements) used in this study

\begin{tabular}{|c|c|c|c|c|}
\hline & Trait 1 & Statement 1 & Trait 2 & Statement 2 \\
\hline 1 & Emo & Get stressed out easily.* & Ext & Don't talk a lot.* \\
\hline 2 & Agr & Feel little concern for others.* & Int & Have a rich vocabulary. \\
\hline 3 & Emo & Am relaxed most of the time. & Int & Have a vivid imagination. \\
\hline 4 & Emo & Worry about things.* & Con & Leave my belongings around.* \\
\hline 5 & Con & Am always prepared. & Int & Have excellent ideas. \\
\hline 6 & Emo & Am easily disturbed.* & Ext & Am the life of the party. \\
\hline 7 & Agr & Insult people.* & Int & Have difficulty understanding abstract ideas.* \\
\hline 8 & Emo & Get upset easily.* & Int & Am not interested in abstract ideas.* \\
\hline 9 & Ext & Keep in the background.* & Con & Pay attention to details. \\
\hline 10 & Ext & Have little to say.* & Int & Am quick to understand things. \\
\hline 11 & Emo & Change my mood a lot.* & Con & Get chores done right away. \\
\hline 12 & Agr & Am interested in people. & Con & Like order. \\
\hline 13 & Emo & Have frequent mood swings.* & Agr & Take time out for others. \\
\hline 14 & Ext & Feel comfortable around people. & Agr & Sympathize with others' feelings. \\
\hline 15 & Con & Make a mess of things.* & Int & Use difficult words. \\
\hline 16 & Emo & Seldom feel blue. & Ext & Start conversations. \\
\hline 17 & Agr & Am not interested in other people's problems.* & Con & Follow a schedule. \\
\hline 18 & Ext & Don't like to draw attention to myself.* & Con & Often forget to put things back in their proper place.* \\
\hline 19 & Ext & Talk to a lot of different people at parties. & Agr & Have a soft heart. \\
\hline 20 & Con & Shirk my duties.* & Int & Do not have a good imagination.* \\
\hline 21 & Emo & Get irritated easily.* & Agr & Am not really interested in others.* \\
\hline 22 & Agr & Feel others' emotions. & Con & Am exacting in my work. \\
\hline 23 & Emo & Often feel blue.* & Int & Spend time reflecting on things. \\
\hline 24 & Ext & Am quiet around strangers.* & Agr & Make people feel at ease. \\
\hline 25 & Ext & Don't mind being the center of attention. & Int & Am full of ideas. \\
\hline
\end{tabular}

Notes: Statements with an asterisk $(*)$ are negative statements; Emo = Emotional Stability (trait number: 1$)$; Ext = Extraversion $(2)$; Agr = Agreeableness (3); Con = Conscientiousness (4); Int = Intellect/Imagination (5); Japanese versions of the statements are available at https://ipip. ori.org/JapaneseBig-FiveFactorMarkers.htm 
data, which we collected along with the response time information.

\section{Data}

We collected data from a sample of 500 Japanese respondents through CrowdWorks, a major online crowdsourcing service in Japan. The sample consists of 232 males and 262 females (the remaining six did not answer the question), and their ages range from their 20 s to their 70 s. The survey was conducted in an online survey environment that we developed with the jsPsych library (de Leeuw, 2015). Online informed consent was obtained from all participants. After data collection, the observations from one respondent were eliminated owing to system trouble. As a result, we applied the proposed model and Thurstonian IRT model to the data with a sample size of 499. In addition, approximately $0.1 \%$ of responses for which the response times were shorter than $300 \mathrm{~ms}$ were listwise deleted. Woodworth and Schlosberg (1954, Chapter 2) indicate that the minimum response time (they use the term latency) for simple visual tasks is approximately $180 \mathrm{~ms}$. However, because the cognitive comparison task of the current study requires a higher cognitive load, more time would be required to answer the task used in this study. Therefore, we set the lower cutoff to $300 \mathrm{~ms}$.

The items we used originate from the Japanese version of the Big-Five factor marker questionnaire (Apple \& Neff, 2012). This scale is intended to measure the Big-Five traits, which are emotional stability, extraversion, agreeableness, conscientiousness, and intellect/imagination. Each trait is measured by 10 statements, which add up to a total of 50 statements. In order to rearrange them into the $2 \mathrm{MFC}$ format, we constructed 25 pairs of items from the 50 statements without duplication. The pairs were carefully designed in order to maintain balance among pairs of traits and to include pairs of statements that are keyed both in the same and opposite directions. Table 3 summarizes the resultant (English-translated) items used in this study. Respondents were required to select the statement of the pair that better represents themselves for all 25 items. The order of the items and the order of statements in each item were randomized across respondents.

\section{Parameter estimation}

We comparatively estimated the parameters for two models: the Thurstonian IRT model, which only uses the item responses as observed variables, and the proposed model, which uses both the item responses and their response times as observed variables. For both models, the number of MCMC iterations per chain was 10,000, half of which were discarded as warm-up samples. The priors used in the
Thurstonian IRT model were a subset of the priors for the proposed model as follows:

$$
\begin{array}{lll}
\beta_{j_{k}} \sim t_{[0, \infty)}(4,0,2.5), & \eta_{i} \sim \operatorname{MVN}(\mathbf{0}, \Sigma), \\
\alpha_{j} \sim N(0,2.5), & \Sigma \sim L K J \operatorname{Corr}(1) .
\end{array}
$$

\section{Results}

Table 4 summarizes the posterior means of the item parameters estimated by both models. Apparently, the point estimates from the Thurstonian IRT model are several times larger than those of the proposed model. A major reason for this is that there exist three types of parameters relates to the discriminability $\left(\beta_{j_{k}}, \xi_{j}\right.$, and $\left.\gamma_{i}\right)$ in the proposed model. In fact, the item parameters obtained by the proposed model become similar to those obtained by the Thurstonian IRT model when they are divided by $\xi_{j}$. This is because all

\begin{tabular}{|c|c|c|c|c|c|c|c|}
\hline \multirow[b]{2}{*}{ item } & \multicolumn{3}{|c|}{ Thurstonian IRT model } & \multicolumn{4}{|c|}{ Proposed model } \\
\hline & $\alpha_{j}$ & $\left|\beta_{j_{1}}\right|$ & $\left|\beta_{j_{2}}\right|$ & $\alpha_{j}$ & $\left|\beta_{j_{1}}\right|$ & $\left|\beta_{j_{2}}\right|$ & $\xi_{j}$ \\
\hline 1 & -0.728 & 2.334 & 1.681 & -0.227 & 0.761 & 0.511 & 0.308 \\
\hline 2 & 1.512 & 1.173 & 1.199 & 0.368 & 0.295 & 0.335 & 0.259 \\
\hline 3 & 1.286 & 1.043 & 1.270 & 0.416 & 0.274 & 0.399 & 0.296 \\
\hline 4 & -1.899 & 1.142 & 1.847 & -0.555 & 0.279 & 0.462 & 0.307 \\
\hline 5 & -1.099 & 1.481 & 1.900 & -0.265 & 0.387 & 0.433 & 0.288 \\
\hline 6 & -1.978 & 0.314 & 1.964 & -0.679 & 0.267 & 0.577 & 0.313 \\
\hline 7 & 0.844 & 0.611 & 0.271 & 0.229 & 0.173 & 0.094 & 0.243 \\
\hline 8 & -1.505 & 0.994 & 0.248 & -0.462 & 0.347 & 0.084 & 0.249 \\
\hline 9 & 0.403 & 1.946 & 0.302 & 0.100 & 0.724 & 0.121 & 0.308 \\
\hline 10 & 0.494 & 1.783 & 0.287 & 0.133 & 0.508 & 0.176 & 0.277 \\
\hline 11 & -0.186 & 1.024 & 1.689 & -0.059 & 0.332 & 0.426 & 0.278 \\
\hline 12 & 0.550 & 2.983 & 3.827 & 0.143 & 0.490 & 0.762 & 0.267 \\
\hline 13 & -0.316 & 1.274 & 0.893 & -0.108 & 0.384 & 0.251 & 0.258 \\
\hline 14 & 1.993 & 1.199 & 1.140 & 0.570 & 0.229 & 0.272 & 0.261 \\
\hline 15 & 0.132 & 0.222 & 0.106 & 0.028 & 0.097 & 0.043 & 0.304 \\
\hline 16 & 0.864 & 1.907 & 1.996 & 0.218 & 0.422 & 0.488 & 0.231 \\
\hline 17 & 1.060 & 0.973 & 0.561 & 0.324 & 0.342 & 0.141 & 0.247 \\
\hline 18 & -0.572 & 0.980 & 1.813 & -0.168 & 0.240 & 0.443 & 0.254 \\
\hline 19 & 2.161 & 1.304 & 0.858 & 0.694 & 0.348 & 0.271 & 0.244 \\
\hline 20 & 0.102 & 1.325 & 1.669 & 0.026 & 0.327 & 0.371 & 0.247 \\
\hline 21 & -1.210 & 1.246 & 1.353 & -0.429 & 0.464 & 0.388 & 0.291 \\
\hline 22 & -0.263 & 0.219 & 0.320 & -0.084 & 0.092 & 0.121 & 0.287 \\
\hline 23 & -0.035 & 1.100 & 0.581 & -0.054 & 0.257 & 0.189 & 0.244 \\
\hline 24 & -1.352 & 1.872 & 0.241 & -0.407 & 0.591 & 0.074 & 0.288 \\
\hline 25 & 0.522 & 0.812 & 1.526 & 0.169 & 0.193 & 0.385 & 0.270 \\
\hline
\end{tabular}

Table 4 Posterior means of the item parameter values in the Thurstonian IRT and proposed models

Note: With regard to $\beta$, absolute values are displayed. In parameter estimation, $\beta$ is restricted to be positive. The estimated values for keyed items were manually sign-inverted afterwards 


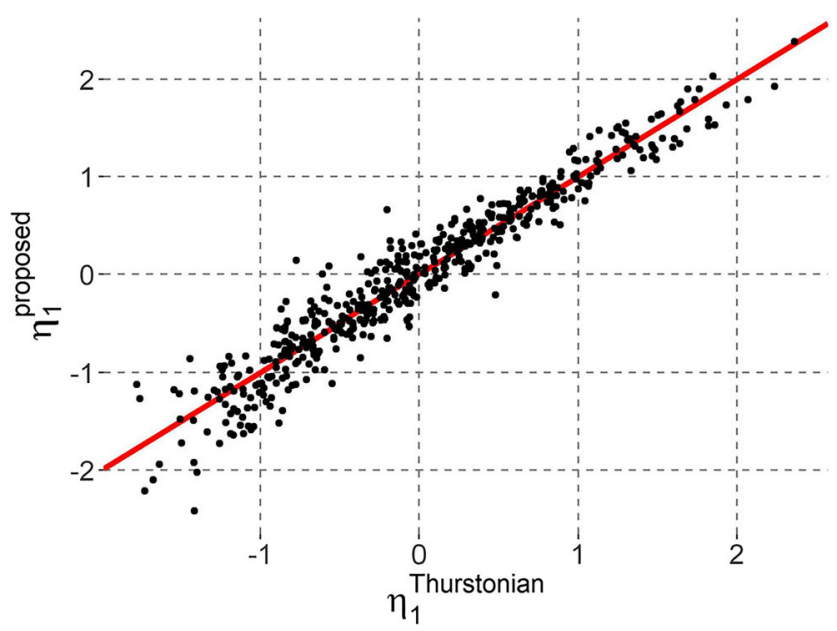

Fig. 2 Scatter plot between the $\eta_{i}$ estimates from the Thurstonian IRT and proposed models with regard to trait 1 (extraversion). Red line indicates the line of $y=x$

values of $\gamma_{i}$ follow $L N(0,1)$ a priori, and the expected value of a random variable that follows $L N(0,1)$ is 1 .

The correlations between the estimates from the two models are .995 for $\alpha_{j}$ and .902 for $\beta_{j_{k}}$. Therefore, it would be appropriate to consider that the item parameters of the proposed model have similar interpretations as those of the Thurstonian IRT model.

Figure 2 shows a scatter plot between the Thurstonian IRT and proposed models in terms of the first trait estimates $\eta_{i 1}$, which correspond to the extraversion trait. The first trait was chosen for illustration purposes, and similar tendencies were observed for all five traits. Table 5 summarizes the correlations between the respondent parameter estimates, both within and between the models. All of the between-model correlations for the same trait are larger than .95, which can be interpreted that very similar respondent parameter estimates are obtained between the two models. Moreover, as for the correlations between traits within a model, similar correlation patterns are obtained for both models, except that they are slightly smaller for the proposed model.

Figure 3 shows the posterior distribution of $\xi_{j}$ for each item. A clear between-item difference can be seen in this figure. In order to check their validity, we calculated the correlation between the posterior means of $\xi_{j}$ and the item mean of the readability scores of each statement. The readability score, which quantifies the difficulty in reading the sentence, was computed by jReadability (Hasebe \& Lee, 2015, https://jreadability.net/sys/);. The resulting correlation was .539 (95\% CI [.162, .751]). This positive correlation can be evidence that $\xi_{j}$ reflects empirical item characteristics such as the reading difficulty.

Figure 4 shows two scatter plots between the boundaryrelated parameter estimates $\left(\gamma_{i}\right.$ and $\left.\xi_{j}\right)$ and the mean response time. It can be seen that there exist strong associations between the two quantities; the obtained correlation coefficients were .929 and -.716 , respectively. These strong relationships between the observed response time and the parameter estimates are consistent with the results of Ratcliff, Thompson, and Mckoon (2015), Tuerlinckx, Molenaar, and Maas (2016). As shown in Eq. 11, the boundary of the diffusion process becomes larger according to the increase in $\gamma_{i}$. This means that a respondent with a higher value of $\gamma_{i}$ needs more information accumulation to respond to an item. As a result, such a respondent tends to have a longer response time. This relationship is properly reflected in the left panel of Fig. 4. The opposite is true for $\xi_{j}$, which is the denominator of the boundary.

Table 5 Estimated correlation matrix in the real data application

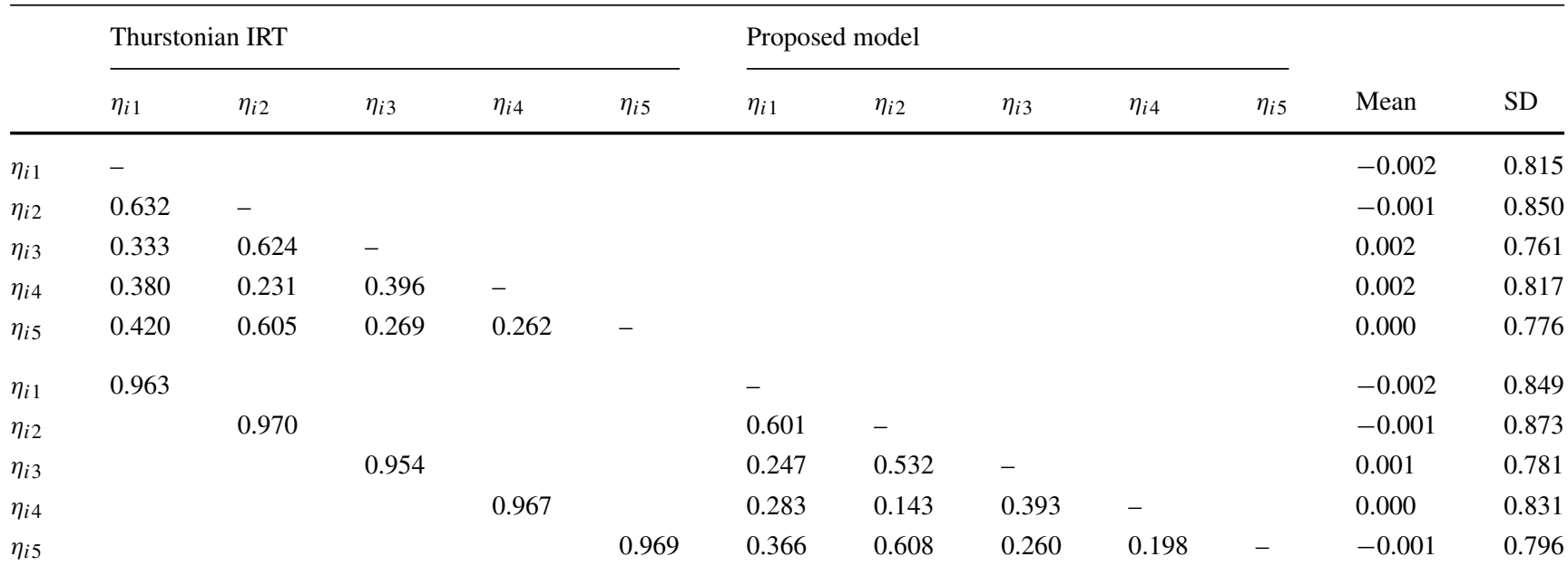

Note: $\eta_{i 1}=$ emotional stability; $\eta_{i 2}=$ extraversion; $\eta_{i 3}=$ agreeableness; $\eta_{i 4}=$ conscientiousness $; \eta_{i 5}=$ intellect $/$ imagination 


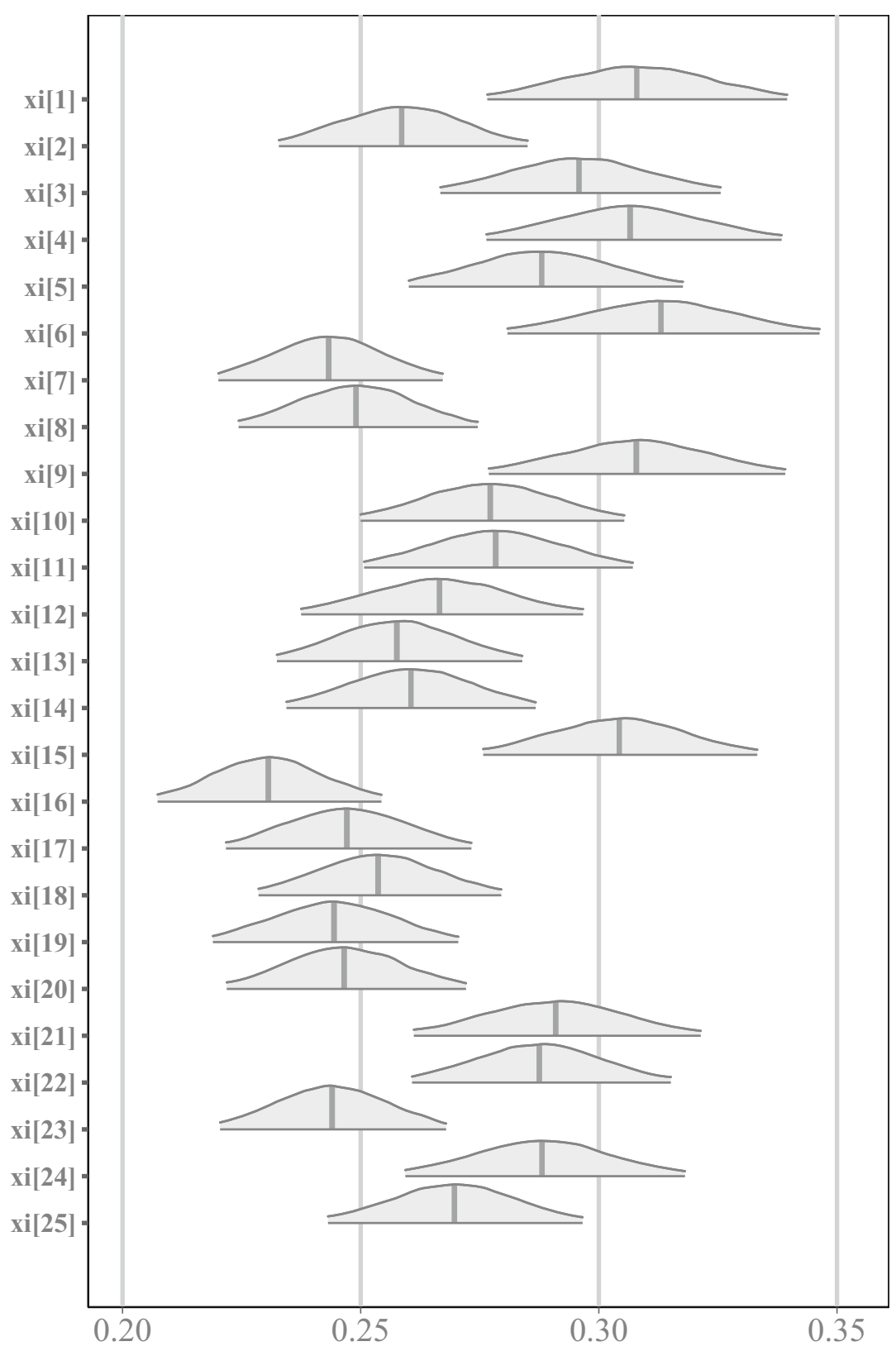

Fig. 3 Posterior distribution of $\xi_{j}$ for each item. The gray area indicates the $95 \%$ CI. The indices of the items correspond to those in Tables 3 and 4

Next, we examined how the estimates from the proposed model reflect the distance-difficulty relationship. For this purpose, Fig. 5 shows scatter plots between the respondent's proximity to the comparison threshold ( $x$ axis), which is given by $\beta_{j_{1}} \eta_{i m_{j_{1}}}-\beta_{j_{2}} \eta_{i m_{j_{2}}}-\alpha_{j}$, and the observed response time ( $y$ axis). In the original diffusion IRT model, the expected response time is given by (Tuerlinckx et al., 2016)

$E\left(t_{i j}\right) \approx \tau_{j}+\frac{1}{2\left|\theta_{i}-b_{j}\right|} \frac{\gamma_{i}}{\xi_{j}}$
From this formula, we can easily obtain the expected response time for the proposed model, i.e.,

$$
E\left(t_{i j}\right) \approx \tau_{j}+\frac{1}{2\left|\beta_{j_{1}} \eta_{i m_{j_{1}}}-\beta_{j_{2}} \eta_{i m_{j_{2}}}-\alpha_{j}\right|} \frac{\gamma_{i}}{\xi_{j}} .
$$

It is seen in the left panel of Fig. 5, which shows the estimates from the Thurstonian IRT model, that the response time tends to be large when the latent positions of the respondent and item parameters are close to each other. This is no surprise given that the distance-difficulty relation is wellknown in personality measurement. However, this tendency 

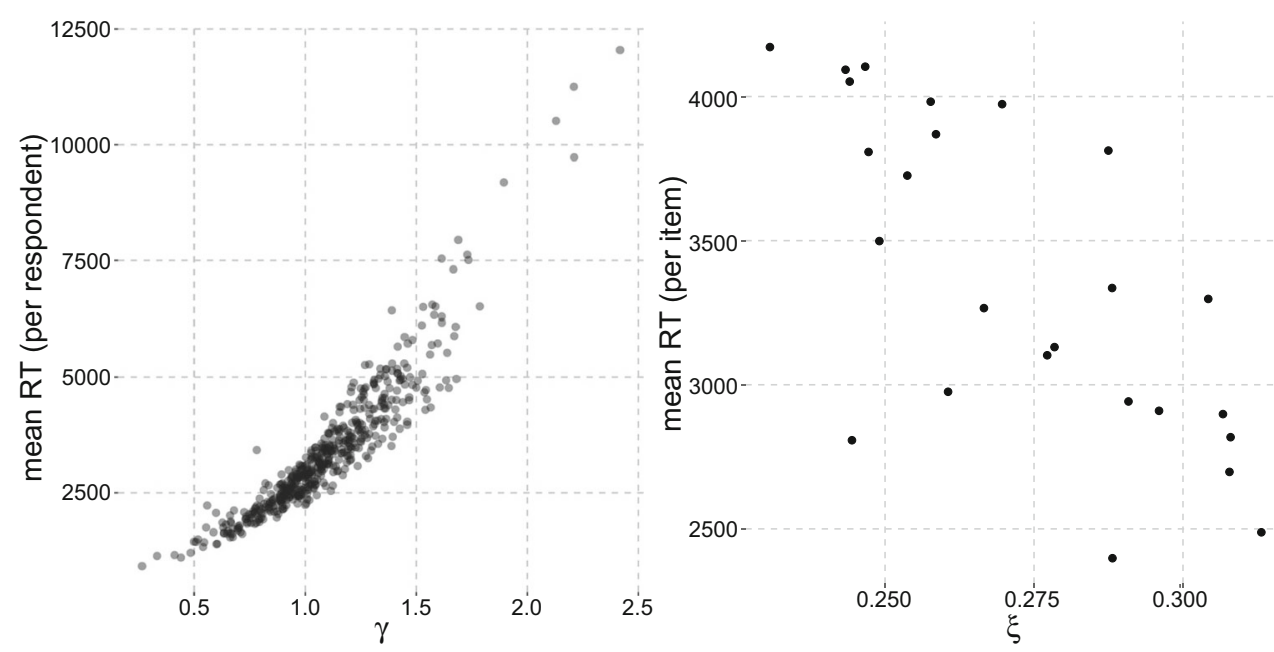

Fig. 4 Left panel $=$ scatter plot between the estimate $\gamma_{i}$ and the mean response time for each respondent. Right panel = scatter plot between the estimate of $\xi_{j}$ and the mean response time for each item

is more clearly evident when the parameters are estimated by the proposed model (right panel). This is because the proposed model explicitly accounts for the distancedifficulty relationship by separating the respondent and item factors that affect the response time, such as the psychological traits to be measured and the item complexity.

Lastly, we examined how the response time information is utilized for estimating $\boldsymbol{\eta}_{i}$ in the proposed model. In the proposed model, the parameter $\boldsymbol{\eta}_{i}$ is estimated on the basis of both the item response and response time, whereas in the existing Thurstonian IRT model, the response time information is not used. In order to examine the unique effect of the response time in the proposed model, we first calculated the mean signed standardized response time (MSSRT) for each respondent and each trait by the following procedure:

1. Prepare all 10 item responses and response times (RTs) for each statement of a trait.

2. Standardize the response time using the whole-sample mean and standard deviation of the item. This yields the standardized RT.

3. When a statement is positively keyed and not chosen or when it is negatively keyed and chosen, make the sign of its standardized RT negative. This yields the signed standardized RT.
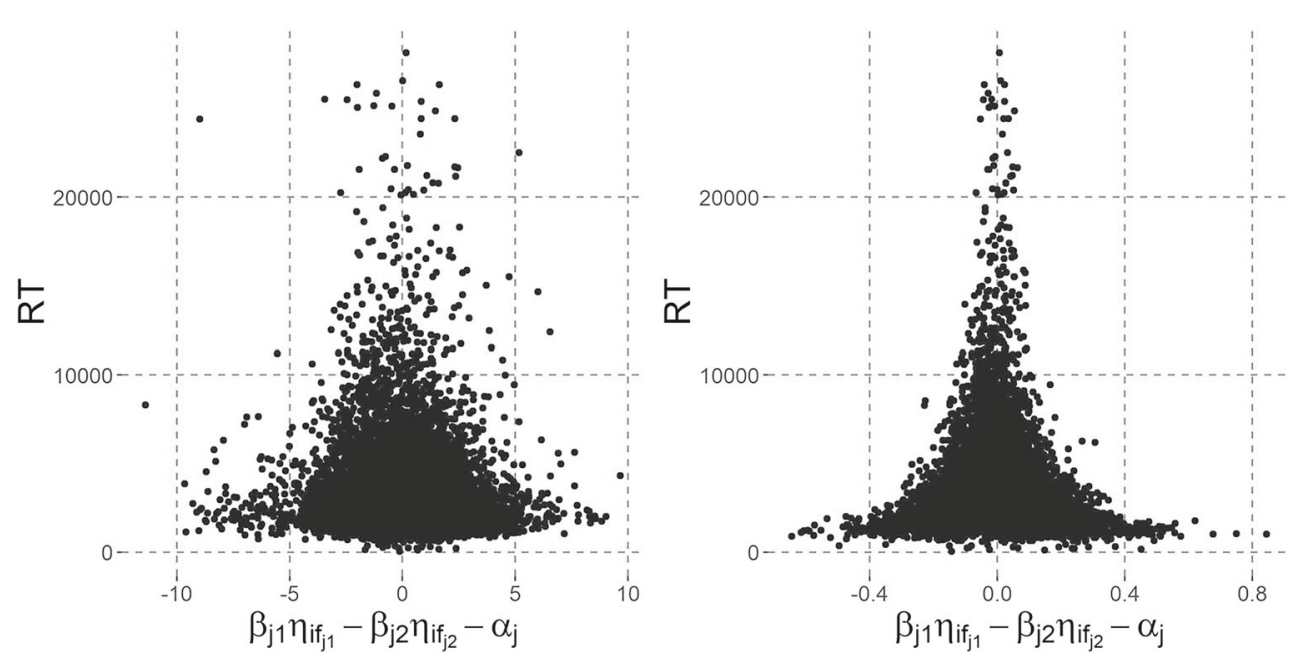

Fig. 5 Scatter plots between the latent respondent position relative to the item parameters ( $x$ axis) and the observed response time ( $y$ axis). Left panel $=$ results for the Thurstonian IRT model. Right panel $=$ results for the proposed model $\left(y\right.$-axis values are divided by $\gamma_{i} / \xi_{j}$ in order to adjust the scale to the left panel) 


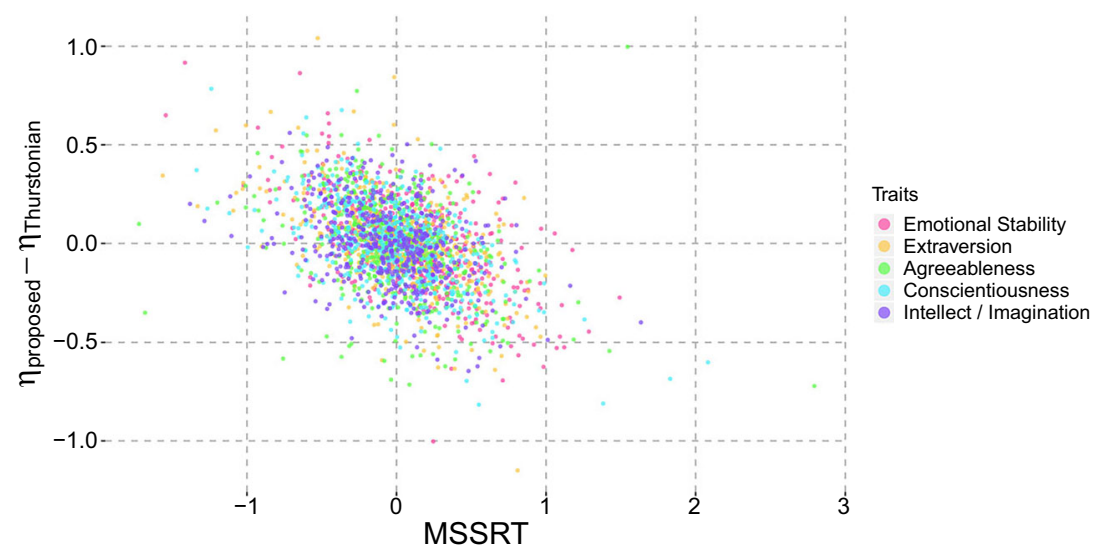

Fig. 6 Scatter plot of the mean signed standardized response time ( $x$ axis) and the difference of trait estimates $\eta_{i}$ between the proposed model and the Thurstonian IRT model ( $y$ axis)

4. Finally, compute the mean of the signed standardized response time for all 10 items. This is the MSSRT.

If the statement that represents a trait is positively keyed and chosen, the signed standardized RT becomes smaller when it is quickly chosen. If it is positively keyed but not chosen, the signed standardized RT becomes smaller when it takes long time to decide not to choose it. The opposite is true when the statement is negatively keyed. That is, the signed standardized RT becomes smaller if a negatively keyed statement is slowly chosen or quickly avoided.

Here, a possible relationship to be found would be the following. For a smaller MSSRT, the estimate obtained by the proposed model tends to be larger compared to that of the Thurstonian IRT model. In other words, a negative correlation would be expected between the MSSRT and the difference of $\boldsymbol{\eta}_{i}$ estimates between the proposed model and the Thurstonian IRT model. Figure 6 shows the scatter plot of the MSSRT and the difference between the two model estimates of $\boldsymbol{\eta}_{i}$. A negative correlation is evident between the two quantities, and little difference among traits was found. The overall correlation was -0.483 $(95 \%$ CI $[-.513,-.452])$. This result suggests that the proposed model successfully incorporates the response time information to supplement the estimation of latent traits.

\section{Discussion}

The main objective of this study is to propose and examine the Thurstonian D-diffusion IRT model, which is an extension of the Thurstonian IRT model for 2MFC measurement to incorporate the response time information. To achieve this goal, we base our model on the diffusion IRT model, which is a representative cognitive psychometric model, and reparameterized its parameters to match the idea of the
Thurstonian IRT model. A simulation study has shown that the parameters of the proposed model can be sufficiently recovered in typical application settings. In the application to Big-Five measurement data, several interesting findings have emerged. The obtained item parameter estimates from the proposed model were similar to those of the Thurstonian IRT model, except for the theoretically expected scale differences. As for the respondent parameters $(\eta)$, the estimates of the proposed model were also similar to those of the Thurstonian IRT, but there is a difference, which was explained by the distance-difficulty relationship. Because this relationship is believed to be generally applicable in personality measurement, we believe that personality estimation based on the proposed Thurstonian D-diffusion IRT model should be meaningful.

When one trait to be measured is clearly dominant over others for a respondent, this respondent is expected to rapidly choose the statements of this trait. In this way, the proposed model should be able to reflect the degree of dominance of one trait over others. Such usage of response time information is not possible for existing Thurstonian IRT models that only use the item response as observations.

According to Bertling and Weeks $(2018$, p. 328) the motivation to utilize the response time information in conjunction with the item response can be classified into two types. The first is "to obtain more accurate proficiency level estimates," and the second is "to estimate examinee performance on a separate latent trait." Although our situation may be different in that the current study considers personality measurement while Bertling and Weeks (2018) considered ability measurement, we believe that the proposed model is closer to the latter case. That is, the proposed Thurstonian D-diffusion IRT model estimates the item and respondent parameters on the basis of psychological theory models of comparative judgment, the diffusion process, and the distance-difficulty relationship. As a 
result, the obtained latent traits would be well-isolated from the effects of factors such as the respondents' deliberateness and item length. Moreover, according to Kahneman (2011), comparative judgment, which involves a slower and more deliberative process, better reflects the cognitive component than single evaluations, which often reflect the intensity of an unstable emotional response. Meanwhile, in the diffusion model, the discrimination parameter correspond to the degree of response caution (boundary); a response that has a longer response time better reflects one's latent trait. Such a correspondence between these two theories suggests the relevance of this study's contribution to extend the diffusion model for Thurstonian judgment.

By introducing the approach of cognitive psychometrics, the parameters of the proposed model can quantify different cognitive subskills separately, such as the speed of information uptake and the degree of response caution. This is in contrast to the classical latent variable in psychometrics, which may be an unknown composite of cognitive processes (Riefer, Knapp, Batchelder, Bamber, \& Manifold, 2002). Although we have not fully investigated the empirical relations between these parameter estimates and other exogenous variables, we consider such a study based on experiments based on an experiment (e.g., Ratcliff \& Rouder, 1998; Voss, Rothermund, \& Voss, 2004) would be of great interest.

Another advantage of the application of cognitive psychometric models is that they can incorporate the properties of a rather complex measurement environment such as the time pressure. For example, Milosavljevic, Malmaud, Huth, Koch, and Rangel (2010) revealed that when a respondent is under a high time pressure condition, the mean response time becomes shorter than under the low time pressure condition; correspondingly, the boundary parameter estimates in the diffusion model become smaller. For this type of high time pressure, the responses become faster and, at the same time, more inaccurate. The diffusion-based models can appropriately account for such effects of the time pressure (e.g., Voss et al., 2004). Such an explanatory power would be one of the advantages of the application of cognitive psychometric models.

Finally, we note a few limitations of the current study. First, a forced-choice item typically provides lower information than a Likert-scale item. As noted by Brown and Maydeu-Olivares (2013), although a five-point Likertscale item provides four pieces of information, a twoalternative forced-choice item provides only one piece of information. Therefore, in practice, care would be needed when designing the forced-choice test, in terms of the number of items and the number of traits, for example, in order to ensure its reliability. Meanwhile, as we noted in the introduction, one of the major motivations to use the forcedchoice format is to reduce the effect of possible biases that originate from the Likert measurement. Recent studies that compared forced-choice Thurstonian IRT measurement and Likert measurement have found evidence that forced-choice measurement has higher validity, even though its obtained information is smaller (e.g., Guenole, Brown, \& Cooper, 2018; Lee, Joo, \& Lee, 2019). That being said, we have not examined the external validity of the latent traits estimated by the proposed model. Although the obtained parameter estimates are based on psychologically meaningful models, an empirical comparison of the external validity of the trait parameter estimates with existing methods remains an important subject for future study.

In addition, we selected the diffusion model as the base model of the current study. A limitation of the diffusion model is that it is only applicable to items that consist of two statements. When considering more than two statements for an item, we have to use other models as the base model. The linear ballistic accumulation (LBA) model (Brown \& Heathcote, 2008) would be a strong candidate in such a case because "For multiple choices between $N>2$ alternatives, only the LBA has simple-to-use analytic solutions, making it the preferred choice" (Brown \& Heathcote, 2008, p. 173). In fact, the LBA model can be thought to be as popular as the diffusion model in cognitive psychology studies. Recently, Bunji and Okada (2019) have developed an IRT extension of the LBA model. Although this is not a statistically straightforward extension, it would be an important direction for further study to extend the LBA IRT model to incorporate the response time information.

\section{References}

Apple, M. T., \& Neff, P. (2012). Using Rasch measurement to validate the Big Five factor marker questionnaire for a Japanese university population. Journal of Applied Measurement, 13(3), 276-296.

Batchelder, W. H. (1998). Multinomial processing tree models and psychological assessment. Psychological Assessment, 10(4), 331344. https://doi.org/10.1037/1040-3590.10.4.331.

Bertling, M., \& Weeks, J. P. (2018). Using response time data to reduce testing time in cognitive tests. Psychological Assessment, 30(3), 328-338. https://doi.org/10.1037/pas0000466.

Brown, A. (2010). How item response theory can solve problems of ipsative data Unpublished doctoral dissertation, University of Barcelona, Spain. Retrieved from, http://hdl.handle.net/10803/ 80006.

Brown, A., \& Maydeu-Olivares, A. (2011). Item response modeling of forced-choice questionnaires. Educational and Psychological Measurement, 71(3), 460-502. https://doi.org/10.1177/ 001316441037511.

Brown, A., \& Maydeu-Olivares, A. (2012). Fitting a Thurstonian IRT model to forced-choice data using Mplus. Behavior Research Methods, 44(4), 1135-1147. https://doi.org/10.3758/s13428-0120217-x.

Brown, A., \& Maydeu-Olivares, A. (2013). How IRT can solve problems of ipsative data in forced-choice questionnaires. Psychological Methods, 18(1), 36-52. https://doi.org/10.1037/a0030641. 
Brown, A., \& Maydeu-Olivares, A. (2018). Modelling forced-choice response formats. In Irwing, P., Booth, T., \& Hughes, D. (Eds.) The Wiley handbook of psychometric testing: A multidisciplinary reference on survey, scale and test development (pp. 523-569). Hoboken: Wiley.

Brown, S. D., \& Heathcote, A. (2008). The simplest complete model of choice response time: Linear ballistic accumulation. Cognitive Psychology, 57(3), 153-178. https://doi.org/10.1016/j.cogpsych. 2007.12.002.

Bunji, K., \& Okada, K. (2019). Item response and response time model for personality assessment via linear ballistic accumulation. Japanese Journal of Statistics and Data Science, 2(1), 263-297. https://doi.org/10.1007/s42081-019-00040-4.

Chen, C., Lee, S.-y., \& Stevenson, H. W. (1995). Response style and cross-cultural comparisons of rating scales among East Asian and North American students. Psychological Science, 6(3), 170-175. https://doi.org/10.1111/j.1467-9280.1995.tb00327.x.

Christiansen, N., Burns, G., \& Montgomery, G. (2005). Reconsidering the use of forced-choice formats for applicant personality assessment. Human Performance, 18(3), 267-307. https://doi.org/ 10.1207/s15327043hup1803_4.

Condon, D. M. (2018). The SAPA personality inventory: An empirically-derived, hierarchically-organized self-report personality assessment model. Retrieved from https://psyarxiv.com/sc4p9/.

de Leeuw, J. R. (2015). jsPsych: A JavaScript library for creating behavioral experiments in a Web browser. Behavior Research Methods, 47(1), 1-12. https://doi.org/10.3758/s13428-014-0458-y.

Ferrando, P. J. (2006). Person-item distance and response time: an empirical study in personality measurement. Psicologica:, International Journal of Methodology and Experimental Psychology, 27(1), 137-148. https://doi.org/10.1348/000711009X470740.

Ferrando, P. J., \& Lorenzo-Seva, U. (2010). Acquiescence as a source of bias and model and person misfit: a theoretical and empirical analysis. British Journal of Mathematical and Statistical Psychology, 63(2), 427-448.

Gelman, A. (2006). Prior distributions for variance parameters in hierarchical models (comment on article by Browne and Draper). Bayesian Analysis, 1(3), 515-534.

Guenole, N., Brown, A., \& Cooper, A. J. (2018). Forced-choice assessment of work-related maladaptive personality traits: Preliminary evidence from an application of Thurstonian item response modeling. Assessment, 25(4), 513-526. https://doi.org/10.1177/ 1073191116641181.

Hasebe, Y., \& Lee, J.-H. (2015). Introducing a readability evaluation system for Japanese language education. In Proceedings of the 6th international conference on computer assisted systems for teaching \& learning japanese (CASTEL/J), (pp. 19-22).

He, J., Bartram, D., Inceoglu, I., \& Vijver, F. J. (2014). Van de Response styles and personality traits: A multilevel analysis. Journal of Cross-Cultural Psychology, 45(7), 1028-1045. https://doi.org/10.1177/0022022114534773.

Hicks, L. E. (1970). Some properties of ipsative, normative, and forced-choice normative measures. Psychological Bulletin, 74(3), 167-184. https://doi.org/10.1037/h0029780.

Jackson, D. N., Wroblewski, V. R., \& Ashton, M. C. (2000). The impact of faking on employment tests: Does forced choice offer a solution? Human Performance, 13(4), 371-388. https://doi.org/ 10.1207/S15327043HUP1304_3.

Johnson, C. E., Wood, R., \& Blinkhorn, S. F. (1988). Spuriouser and spuriouser: the ues of ipsative personality tests. Journal of Occupational Psychology, 61, 153-162. https://doi.org/10.1111/j. 2044-8325.1988.tb00279.x.

Kahneman, D. (2011). Thinking, Fast and Slow. New York: Farrar, Straus and Giroux.
Kam, C. C. S., \& Meyer, J. P. (2015). How careless responding and acquiescence response bias can influence construct dimensionality: the case of job satisfaction. Organizational Research Methods, 18(3), 512-541. https://doi.org/10.1177/1094428115571894.

Kong, X. J., Wise, S. L., \& Bhola, D. S. (2007). Setting the response time threshold parameter to differentiate solution behavior from rapid-guessing behavior. Educational and Psychological Measurement, 67(4), 606-619. https://doi.org/10.1177/0013164406294779.

Kuncel, R. B. (1973). Response processes and relative location of subject and item. Educational and Psychological Measurement, 33(3), 545-563. https://doi.org/10.1177/001316447303300302.

Lee, M. D., \& Wagenmakers, E.-J. (2013). Bayesian Cognitive Modeling: A Practical Course. Cambridge: Cambridge University Press.

Lee, P., Joo, S. H., \& Lee, S. (2019). Examining stability of personality profile solutions between Likert-type and multidimensional forced choice measure. Personality and Individual Differences, 142, 1320. https://doi.org/10.1016/j.paid.2019.01.022.

Lewandowski, D., Kurowicka, D., \& Joe, H. (2009). Generating random correlation matrices based on vines and extended onion method. Journal of Multivariate Analysis, 100(9), 1989-2001. https://doi.org/10.1016/j.jmva.2009.04.008.

Likert, R. (1932). A technique for the measurement of attitudes. Archives of Psychology, 22(140), 1-55.

Liu, M., Harbaugh, A. G., Harring, J. R., \& Hancock, G. R. (2017). The effect of extreme response and non-extreme response styles on testing measurement invariance. Frontiers in Psychology, 8, Article 726. https://doi.org/10.3389/fpsyg.2017.00726.

Lord, F. M. (1980). Applications of item response theory to practical testing problems. Hillsdale: Erlbaum.

Milosavljevic, M., Malmaud, J., Huth, A., Koch, C., \& Rangel, A. (2010). The Drift Diffusion Model can account for the accuracy and reaction time of value-based choices under high and low time pressure. Judgment and Decision Making, 5(6), 437-449. https://doi.org/10.2139/ssrn.1901533.

Molenaar, D., \& Bolsinova, M. (2017). A heteroscedastic generalized linear model with a non-normal speed factor for responses and response times. British Journal of Mathematical and Statistical Psychology, 70, 297-316. https://doi.org/10.1111/bmsp.12087.

Molenaar, D., Tuerlinckx, F., \& Maas, H. L. (2015). Van der Fitting diffusion item response theory models for responses and response times using the R package diffIRT. Journal of Statistical Software, 66(4), 1-34. https://doi.org/10.18637/jss.v066.i04.

Navarro, D. J., \& Fuss, I. G. (2009). Fast and accurate calculations for first-passage times in Wiener diffusion models. Journal of Mathematical Psychology, 53(4), 222-230. https://doi.org/10.1016/j. jmp.2009.02.003.

Phelps, L., Schmitz, C. D., \& Boatright, B. (1986). The effects of halo and leniency on cooperating teacher reports using Likert-type rating scales. Journal of Educational Research, 79(3), 151-154. https://doi.org/10.1080/00220671.1986.10885668.

Podsakoff, P. M., MacKenzie, S. B., Lee, J. Y., \& Podsakoff, N. P. (2003). Common method biases in behavioral research: a critical review of the literature and recommended remedies. Journal of Applied Psychology, 88(5), 879-903. https://doi.org/10.1037/ 0021-9010.88.5.879.

Rammstedt, B., \& Farmer, R. F. (2013). The impact of acquiescence on the evaluation of personality structure. Psychological Assessment, 25(4), 1137-1145. https://doi.org/10.1037/a0033323.

Ranger, J., Kuhn, J.-T., \& Szardenings, C. (2016). Limited information estimation of the diffusion-based item response theory model for responses and response times. British Journal of Mathematical and Statistical Psychology, 69(2), 122-138. https://doi.org/10.1111/bmsp.12064. 
Ranger, J., Kuhn, J.-T., \& Szardenings, C. (2017). Analysing model fit of psychometric process models: an overview, a new test and an application to the diffusion model. British Journal of Mathematical and Statistical Psychology, 70(2), 209-224. https://doi.org/10.1111/bmsp.12082.

Rasch, G. (1960). Probabilistic models for some intelligence and attainment tests. Copenhagen, Denmark: Danish Institute for Educational Research.

Ratcliff, R. (1978). A theory of memory retrieval. Psychological Review, 85(2), 59-108. https://doi.org/10.1037/0033-295X.85. 2.59 .

Ratcliff, R., \& McKoon, G. (2008). The diffusion decision model: theory and data for two-choice decision tasks. Neural Computation, 20(4), 873-922. https://doi.org/10.1162/neco.2008.12-06-420.

Ratcliff, R., \& Rouder, J. N. (1998). Modeling response times for two-choice decisions. Psychological Science, 9(5), 347-356. https://doi.org/10.1111/1467-9280.00067.

Ratcliff, R., Thompson, C. A., \& Mckoon, G. (2015). Modeling individual differences in response time and accuracy in numeracy. Cognition, 137, 115-136. https://doi.org/10.1016/j.cognition. 2014.12.004.

Raz, S., Bar-Haim, Y., Sadeh, A., \& Dan, O. (2014). Reliability and validity of the online continuous performance test among young adults. Assessment, 21(1), 108-118. https://doi.org/10.1177/ 1073191112443409.

Riefer, D. M., Knapp, B. R., Batchelder, W. H., Bamber, D., \& Manifold, V. (2002). Cognitive psychometrics: Assessing storage and retrieval deficits in special populations with multinomial processing tree models. Psychological Assessment, 14(2), 184-201. https://doi.org/10.1037/1040-3590.14.2.184.

Salgado, J. F. (2016). A theoretical model of psychometric effects of faking on assessment procedures: Empirical findings and implications for personality at work. International Journal of Selection and Assessment, 24(3), 209-228. https://doi.org/10.1111/ijsa. 12142.

Salgado, J. F., \& Táuriz, G. (2014). The Five-Factor Model, forcedchoice personality inventories and performance: A comprehensive meta-analysis of academic and occupational validity studies. European Journal of Work and Organizational Psychology, 23(1), 3-30. https://doi.org/10.1080/1359432X.2012.716198.

Stan Development Team (2017). Stan modeling language users guide and reference manual, Version 2.17.0. Retrieved from http:// mc-stan.org.

Stone, C. A. (1992). Recovery of marginal maximum likelihood response model: An Evaluation of MULTILOG. Applied Psychological Measurement, 16(1), 1-16. https://doi.org/10.1177/ 014662169201600101.

Thunholm, P. (2001). Social desirability in personality testing of military officers. Military Psychology, 13(4), 223-234. https:// doi.org/10.1207/S15327876MP1304_3.
Thurstone, L. L. (1927). A law of comparative judgment. Psychological Review, 34(4), 273-286.

Tuerlinckx, F., \& De Boeck, P. (2005). Two interpretations of the discrimination parameter. Psychometrika, 70(4), 629-650. https://doi.org/10.1007/s11336-000-0810-3.

Tuerlinckx, F., Molenaar, D., \& van der Maas, H. L. J. (2016). Diffusion-based response-time models. In van der Linden, W. J. (Ed.) Handbook of item response theory: Vol. 1. Models, (pp. 283-300). Boca Raton: CRC Press.

Usami, S., Sakamoto, A., Naito, J., \& Abe, Y. (2016). Developing pairwise preference-based personality test and experimental investigation of its resistance to faking effect by item response model. International Journal of Testing, 16(4), 288-309.

van der Maas, H. L. J., Molenaar, D., Maris, G., Kievit, R. A., \& Borsboom, D. (2011). Cognitive psychology meets psychometric theory: on the relation between process models for decision making and latent variable models for individual differences. Psychological Review, 118(2), 339-356. https://doi.org/10.1037/a0022749.

Vandekerckhove, J. (2014). A cognitive latent variable model for the simultaneous analysis of behavioral and personality data. Journal of Mathematical Psychology, 60, 58-71. https://doi.org/10.1016/j. jmp.2014.06.004.

van Ravenzwaaij, D., \& Oberauer, K. (2009). How to use the diffusion model:, Parameter recovery of three methods: EZ, fast-dm, and DMAT. Journal of Mathematical Psychology, 53(6), 463-473. https://doi.org/10.1177/00131649921969802.

Viswesvaran, C., \& Ones, D. S. (1999). Meta-analyses of fakability estimates: Implications for personality measurement. Educational and Psychological Measurement, 59(2), 197-210. https://doi.org/10.1177/00131649921969802.

Voss, A., Nagler, M., \& Lerche, V. (2013). Diffusion models in experimental psychology: a practical introduction. Experimental Psychology, 60(6), 385-402. https://doi.org/10.1027/1618-3169/ a000218.

Voss, A., Rothermund, K., \& Voss, J. (2004). Interpreting the parameters of the diffusion model: an empirical validation. Memory \& Cognition, 32(7), 1206-1220. https://doi.org/10.3758/BF03196893.

Wang, C., Xu, G., \& Shang, Z. (2018). A two-stage approach to differentiating normal and aberrant behavior in computer based testing. Psychometrika, 83(1), 223-254. https://doi.org/10.1007/s11336016-9525-x.

Wise, S. L., \& Kong, X. (2005). Response time effort: a new measure of examinee motivation in computer-based tests. Applied Measurement in Education, 18(2), 163-183. https://doi.org/10.1207/ s15324818ame1802_2.

Woodworth, R. S., \& Schlosberg, H. (1954). Experimental Psychology. New York: Holt.

Publisher's note Springer Nature remains neutral with regard to jurisdictional claims in published maps and institutional affiliations. 